\title{
EMBEDDINGS OF HARISH-CHANDRA MODULES, n-HOMOLOGY AND THE COMPOSITION SERIES PROBLEM: THE CASE OF REAL RANK ONE
}

BY

DAVID H. COLLINGWOOD

\begin{abstract}
Let $G$ be a connected semisimple matrix group of real rank one. Fix a minimal parabolic subgroup $P=M A N$ and form the (normalized) principal series representations $I_{P}^{G}(U)$. In the case of regular infinitesimal character, we explicitly determine (in terms of Langlands' classification) all irreducible submodules and quotients of $I_{P}^{G}(U)$. As a corollary, all embeddings of an irreducible Harish-Chandra module into principal series are computed. The number of such embeddings is always less than or equal to three. These computations are equivalent to the determination of zero n-homology.
\end{abstract}

1. Introduction. Let $G$ be a connected semisimple matrix group of real rank one. Fix an Iwasawa decomposition $G=K A N$ and a minimal parabolic subgroup $P=M A N$. Let $(\sigma, U)$ be an irreducible $M A$-module. By right translation, the group $G$ acts on the space of smooth functions $f: G \rightarrow U$ satisfying the transformation law: $f(p g)=\left[\sigma(p) \otimes e^{\bar{\rho}}(p)\right] f(g), p \in P, g \in G$ and $\bar{\rho}=$ half-sum of positive restricted roots (counting multiplicities) determined by $P$. Let ind ${ }_{P}^{G}(U)$ denote the Hilbert space completion relative to $\left(f_{1} \mid f_{2}\right)=\int_{K}\left(f_{1}(k), f_{2}(k)\right) d k$. We define

$$
I_{P}^{G}(U)=K \text {-finite vectors in } \operatorname{ind}_{P}^{G}(U) .
$$

The $I_{P}^{G}(U)$ are Harish-Chandra modules with infinitesimal character (cf. §2) and we refer to these as principal series representations.

In view of Casselman's subrepresentation theorem [1], every irreducible HarishChandra module for $G$ may be realized as a submodule of some principal series representation. This leads us to the following problems:

Composition Series Problem. Study the Jordan-Hölder series for $I_{P}^{G}(U)$.

Embedding Problem. Study the embeddings of irreducible Harish-Chandra modules into principal series.

In our setting, the composition series problem has been solved, on the level of the Grothendieck group, cf. [6-8 and 18]. (We use the notation $\mathscr{G}$ to denote the Grothendieck group of virtual characters [17, $(0.3 .16)]$.) This solution relies upon D.

Received by the editors April 26, 1983, and, in revised form, July 25, 1983.

1980 Mathematics Subject Classification. Primary 22E45; Secondary 20 G05.

Key words and phrases. Representations of semisimple Lie groups, embedding theorems, composition series problem, Kazhdan-Lusztig conjectures. 
Vogan's algorithm for computing characters [13] and the Kazhdan-Lusztig conjectures, in the case of regular integral infinitesimal character [15]. In this paper, we undertake a closer investigation of the composition factors for $I_{P}^{G}(U)$. In the case of regular infinitesimal character, we explicitly determine (in terms of Langlands' classification) all irreducible submodules and quotients of $I_{P}^{G}(U)$. We shall refer to this as the fine structure of the composition series for $I_{P}^{G}(U)$. Our main result is

(1.2) THEOREM. Let $I_{P}^{G}(U)$ be a reducible principal series representation, with regular infinitesimal character. The fine structure of the composition series is determined by (4.1)-(4.4). In particular, either $I_{P}^{G}(U)$ decomposes according to Schmid's character identity $[\mathbf{1 0}]$ or $I_{P}^{G}(U)$ has a unique irreducible submodule, a unique irreducible quotient and all intermediary subquotients have trivial extensions with one another.

The proof of (1.2) is broken into the four standard real rank one cases; cf. §3. Our procedure involves a careful analysis of the $\tau$-invariant (which codifies the result of "tensoring an irreducible to the wall") and the $\mathscr{S}$-invariant (which places $\tau$-invariant restrictions on any irreducible submodule of a "standard representation").

Let $V$ be an irreducible Harish-Chandra module for $G$, with regular infinitesimal character. As a corollary to (1.2), we can easily compute the inducing data $U$, for which $V \hookrightarrow I_{P}^{G}(U)$; see $\S 5$. According to Casselman's Frobenius reciprocity formula [1]:

$$
\operatorname{Hom}_{(\mathfrak{g}, K)}\left(V, I_{P}^{G}(U)\right)=\operatorname{Hom}_{M A}\left(H_{0}(\mathfrak{n}, V), U \otimes e^{\bar{\rho}}\right),
$$

where $H_{0}(\mathrm{n}, V)=V / \mathrm{n} V$ is a finite dimensional $M A$-module, the zero $\mathrm{n}$-homology of $V$. By our regularity assumption, $H_{0}(\mathrm{n}, V)$ is a semisimple $M A$-module $[3,(6.6)]$. Thus, computing the embeddings of $V$ is equivalent to explicit determination of $H_{0}(\mathrm{n}, V)$, as an $M A$-module. In $\S 5$, we give the embedding lattice for $G$, which among other things, indicates the number of embeddings of $V$ into principal series. As an interesting consequence, this number is always less than or equal to three. (The case of a unique embedding was addressed in [2].)

The results in this paper have several consequences concerning the asymptotic behavior of matrix coefficients, only one of which we mention here. Let us assume that $I_{P}^{G}(U)$ satisfies $U=W \otimes e^{\nu}, \nu$ positive on a. D. Miličić [9] has shown that $I_{P}^{G}(U)$ contains a unique irreducible quotient, having asymptotic behavior which is strictly worse than that of any other subquotient of $I_{P}^{G}(U)$. This suggests the possibility of filtering a Jordan-Hölder series for $I_{P}^{G}(U)$, using asymptotic growth. Such a filtration is possible when $G=\operatorname{Spin}(n, 1), \operatorname{SU}(n, 1)$. However, our computations in $\operatorname{SP}(\mathrm{n}, 1)$ and $F_{4}$ illustrate: there exists $I_{P}^{G}(U)$ containing a unique nontempered irreducible quotient, a unique nontempered irreducible submodule and a discrete series subquotient. Consequently, there does not exist (an obvious) asymptotic filtration on the Jordan-Hölder series, in general.

As a final note, we remark on the case when $I_{P}^{G}(U)$ is reducible and has singular infinitesimal character. Using "translation functors to the walls"-as is fully described in $[17,(8.7)]$ - the problem of computing the composition factors of $I_{P}^{G}(U)$ is reduced to computations in the case of regular infinitesimal character; the main 
difficulty involves careful "bookkeeping". These remarks, (3.1) and $\tau$-invariant computations, show that $I_{P}^{G}(U)$ has at most two composition factors. (This computation is left to the reader). In the case of length two, the fine structure is either immediate from (2.7) (in the case when $U=W \otimes e^{\nu}, \operatorname{Re} \nu \neq 0$ ), or a direct sum (in the case when $I_{P}^{G}(U)$ belongs to the unitary principal series).

ACKNOWLEDGEMENTS. The approach and results of this paper are a by-product of the authors discussions with David Vogan, concerning the fine structure of composition series for $\mathrm{SP}(2,1)$. The computation of composition factors for $F_{4}$ is due to $\mathrm{H}$. Kraljević and W. Silva [8]. The author also wishes to thank the referee for suggestions on improving the exposition and clarity of the paper.

2. Preliminaries. Throughout this paper, $G$ will denote a connected semisimple matrix group of real rank one. In view of the classification of real forms, $G$ is finitely covered by one of the following groups:

$$
\begin{aligned}
& \operatorname{SL}(2, \mathbf{R}) ; \operatorname{Spin}(n, 1)(n \geqslant 3) ; \operatorname{SU}(n, 1)(n \geqslant 2) ; \operatorname{SP}(n, 1)(n \geqslant 2) ; \\
& F_{4}=\text { analytic group corresponding to the real form } \mathfrak{f}_{4(-2)} .
\end{aligned}
$$

Fix a maximal compact subgroup $K \subseteq G$, which arises as the fixed point set of an involutive automorphism $\theta$ of $G$. Fix an Iwasawa decomposition $G=K A N$, a maximally split $\theta$-stable Cartan subgroup $H=T A(T=K \cap H)$ and a minimal parabolic subgroup $P=M A N$. The real Lie algebras of $G, K, A, N$, etc. are denoted by $\mathfrak{g}_{0}, \mathfrak{f}_{0}, \mathfrak{a}_{0}, \mathfrak{n}_{0}$, etc., respectively. Dropping the subscript “ 0 " indicates complexification. Equip $\mathfrak{g}_{0}$ with a nondegenerate bilinear form $(\mid)$, which is $\operatorname{Ad}(G)$-invariant. If $\mathfrak{g}_{0}=\mathfrak{f}_{0} \oplus \mathfrak{g}_{0}$ is the Cartan decomposition of $\mathfrak{g}_{0}$ corresponding to $\theta$, then we further assume that $(\mid)$ restricted to $\mathfrak{g}_{0}$ (resp. $\mathfrak{f}_{0}$ ) is positive (resp. negative) definite. We use the notation ( $\mid$ ) to denote the restriction of (1) to any $\theta$-stable subspace of $\mathfrak{g}_{0}$, as well as the transported form on the complexified dual.

If $\mathfrak{g}_{0} \neq \mathfrak{S} \mathfrak{o}(2 n+1,1)(n \geqslant 1)$, then rank $G=\operatorname{rank} K$. In this case, $G$ contains a compact Cartan subgroup $B$, with Lie algebra $\mathfrak{b}_{0}$. If $\mathfrak{g}_{0}=\mathfrak{b} \mathfrak{o}(2 n+1,1)(n \geqslant 1)$, then $G$ has one conjugacy class of Cartan subgroups.

By a Harish-Chandra module for $G$, we shall mean a module $V$ over the universal enveloping algebra $\mathfrak{U}(\mathfrak{g})$, together with an action of $K$, such that:

(a) The representation of $K$ is an algebraic direct sum of irreducible finite dimensional continuous representations, each isomorphism class occurring with finite multiplicity.

(b) The representation of $\mathfrak{f}$, as a subalgebra of $\mathfrak{g}$, coincides with the differential of the $K$-action.

(c) $V$ has finite length as a $\mathfrak{U}(\mathfrak{g})$-module.

According to a result of Casselman-Prischepionok [1], any Harish-Chandra module $V$ for $G$ globalizes to $G$. That is, there exists a finite length admissible representation $\pi$ of $G$ on a Hilbert space $V_{\pi}$, such that $V$ may be identified with the $K$-finite vectors in $V_{\pi}$. (This globalization procedure is highly noncanonical.)

Fix an abstract Cartan subalgebra $\mathfrak{h}_{a} \subseteq \mathfrak{g}$. Denote by $\Phi_{a}$ the roots of $\mathfrak{h}_{a}$ in $\mathfrak{g}$, and let $W\left(\Phi_{a}\right)=$ Weyl group of the root system $\Phi_{a}$. We now recall the Harish-Chandra 
parametrization of infinitesimal characters [3]: Let $\supsetneqq(\mathfrak{g})=$ center of $\mathfrak{U}(\mathfrak{g})$. Using the Harish-Chandra isomorphism, every algebra homomorphism $\chi: \nexists(\mathfrak{g}) \rightarrow \mathbf{C}$, such that $\chi(1)=1$, is of the form

$$
\chi_{\gamma}: 3(\mathfrak{g}) \rightarrow \mathbf{C},
$$

for some $\gamma \in \mathfrak{h}_{a}^{*}$. For our purposes, a precise definition of $\chi_{\gamma}$ is unnecessary. We need only recall

$$
\chi_{\gamma}=\chi_{\gamma^{\prime}} \Leftrightarrow \gamma \text { and } \gamma^{\prime} \text { lie in the same } W\left(\Phi_{a}\right) \text {-orbit. }
$$

Every irreducible Harish-Chandra module has an infinitesimal character, as does any principal series representation. We say $\chi_{\gamma}$ is regular (resp. integral) if $(\gamma \mid \alpha) \neq 0$ for all $\alpha \in \Phi_{a}\left(\operatorname{resp} .2(\gamma \mid \alpha) /(\alpha \mid \alpha) \in \mathbf{Z}\right.$ for all $\left.\alpha \in \Phi_{a}\right)$.

Fix a $\theta$-stable Cartan subgroup

$$
H_{a}=T_{a} A_{a} \quad\left(H_{a} \cap K=T_{a} \text { and } A_{a}=\exp \left(\mathfrak{h}_{a, 0} \cap \mathfrak{F}_{0}\right)\right)
$$

and let $\gamma$ be a regular element of $\mathfrak{h}_{a}^{*}$. We define

(2.2) $\Phi^{+}(\gamma)=\left\{\alpha \in \Phi_{a}: \operatorname{Re}(\gamma \mid \alpha)>0\right.$, or $\operatorname{Re}(\gamma \mid \alpha)=0$ and $\left.\operatorname{Im}(\gamma \mid \alpha)>0\right\}$;

which is a system of positive roots for $\Phi_{a}$, having simple roots denoted by $\Delta^{+}(\gamma)$. If $M_{a} A_{a}$ is the Langlands decomposition of the centralizer of $A_{a}$ in $G$, then $T_{a}$ becomes a Cartan subgroup of $M_{a}$, leading to the root system $\Phi\left(\mathrm{t}_{a}\right)$.

Having specified an Iwasawa decomposition $\mathfrak{g}=\mathfrak{f} \oplus \mathfrak{a} \oplus \mathfrak{n}$, we obtain a system of positive restricted roots $\Sigma^{+}$. Choose a compatible positive root system $\Phi^{+}$(here, $\mathfrak{h}_{a}=\mathfrak{h}$ in the above discussion):

$$
\alpha \in \Phi^{+},\left.\quad \alpha\right|_{\mathfrak{a}} \neq\left. 0 \Rightarrow \alpha\right|_{\mathfrak{a}} \in \Sigma^{+} .
$$

We obtain $\Phi^{+}(\mathrm{t})=\Phi^{+} \cap \Phi(\mathrm{t})$; recall, roots in $\Phi(\mathrm{t})$ may be identified with those elements of $\Phi$ vanishing on a.

(2.3) In the event $G$ contains a compact Cartan $\operatorname{subgroup} B$, we fix a positive system of roots in $\mathfrak{b}^{*}$, denoted $\Psi$.

We conclude this section by recalling Langlands' classification, as formulated in $[13,(4.2)]$.

(2.4) Definition. Fix a $\theta$-stable Cartan subgroup $H_{a}=T_{a} A_{a}$. A regular character of $H_{a}$ is an ordered pair $(\Gamma, \gamma)$, having the following properties:

(a) $\Gamma$ is a character of $H_{a}$;

(b) $\gamma \in \mathfrak{h}_{a}^{*}$ is $\Phi_{a}$-regular;

(c) $d \Gamma=\gamma+\delta_{\mathfrak{m}_{a}}-2 \delta_{\mathfrak{m}_{a}} \cap \mathfrak{l}$, where $\delta_{\mathfrak{m}_{a}}$ is the obvious half-sum of positive roots;

(d) if $H_{a}=H$, then $\operatorname{Re}\left(\gamma_{\mid \mathrm{a}} \mid \alpha\right) \geqslant 0$, for $\alpha \in \Sigma^{+}$.

Let $\rho$ denote the half-sum of positive roots in $\Phi^{+}$. We denote by $\hat{H}_{a}$ the collection of all regular characters of $H_{a}$ and set

$$
\hat{H}_{a, \rho}=\left\{(\Gamma, \gamma) \in \hat{H}_{a}: \chi_{\gamma}=\chi_{\rho}\right\} .
$$

We will abuse notation and write $\gamma \in \hat{H}_{a}$; cf. (3.3). If $\gamma \in \hat{B}$, then according to Harish-Chandra, $\gamma$ corresponds to a discrete series representation $\pi_{G}(\gamma)$. If $\gamma \in \hat{H}$, then $\Gamma$ determines an irreducible finite dimensional representation of the compact group $M$, call it $W_{\gamma}$. Further, $e^{\nu}=\exp \left(\left.\gamma\right|_{\mathrm{a}}\right)$ is a character of $A$. Recall (1.1) and put

$$
U_{\gamma}=W_{\gamma} \otimes e^{\nu}, \quad \pi(\gamma)=I_{P}^{G}\left(U_{\gamma}\right) \text {. }
$$


The representations $\pi(\gamma)$, attached to $\hat{H}_{a}$, are called standard representations. These are Harish-Chandra modules with infinitesimal character $\chi_{\gamma}$.

(2.7) Theorem (HaRish - ChandRa, LANglands, Miličić [13, §4]). A standard representation $\pi(\gamma)$ contains a unique irreducible quotient, to be denoted $\bar{\pi}(\gamma)$. Every irreducible Harish-Chandra module for $G$, with regular infinitesimal character, is isomorphic to some $\bar{\pi}(\gamma)$.

(2.8) REMARK. In this paper, we have adopted the "quotient viewpoint" in Langlands' classification. This allows the most direct application of results in $[7,8$ and 11].

3. The algorithm. Our goal is to compute the fine structure of the composition series for the principal series representations. In this section, the problem is reduced to the case of trivial infinitesimal character. In this setting, we describe an algorithm for computing the fine structure of the composition series; cf. [2, \$7]. Along the way, we recall the notion of the $\tau$-invariant, the Kazhdan-Lusztig conjectures and introduce the $\mathscr{S}$-invariant of a standard representation.

(3.1) THEOREM. If $I_{P}^{G}(U)$ is a reducible principal series representation for $G$, then $I_{P}^{G}(U)$ has integral infinitesimal character.

Proof. For the classical groups, see [6,7 and 18]. The case of $F_{4}$ proceeds as for $\mathrm{SP}(n, 1)$; relying upon the major result in [12]. Q.E.D.

In case $G$ is covered by $\operatorname{SL}(2, \mathbf{R})$, the results of this paper are very well known; e.g. see $[17,1.3 .3]$. We now make

(3.2) Assumption. For the remainder of this paper, $G$ is assumed to be one of the following groups: $\operatorname{Spin}(n, 1),(n \geqslant 3)$; $\mathrm{SU}(n, 1),(n \geqslant 2)$; $\operatorname{SP}(n, 1),(n \geqslant 2)$ or $F_{4}$.

(3.3) REMARK. This assumption is no loss of generality, since any group of the type considered in $\$ 2$ will be covered by a group of the type in (3.2). As a further consequence of (3.2), $M$ is connected [5]. This permits an infinitesimal parametrization of $\hat{H}_{a}$.

Let $V$ be a Harish-Chandra module for $G$ with infinitesimal character $\chi_{\gamma}, \gamma \in \mathfrak{h}_{a}^{*}$. Write $\Lambda_{a}$ for the lattice of $\mathfrak{h}_{a}$-weights of finite dimensional representations of $G$. Given $\mu \in \Lambda_{a}$, let $F_{\mu}$ be the irreducible finite dimensional representation of $G$ of extremal weight $\mu$. Let $\mu_{1}, \mu_{2} \in \Lambda_{a}$ and define the translation functors by

$$
\psi_{\gamma+\mu_{2}}^{\gamma+\mu_{1}}(V)=p_{\gamma+\mu_{2}}\left(F_{\mu_{2}-\mu_{1}} \otimes p_{\gamma+\mu_{1}}(V)\right) \text {. }
$$

Here, for $\eta \in \mathfrak{h}_{a}^{*}$,

$$
p_{\eta}(X)=\left\{x \in X \text { : for some } n>0 \text {, and all } Z \in 3(g),\left(Z-\chi_{\eta}(Z)\right)^{n} x=0\right\} .
$$

Further, suppose that $\gamma$ is regular for $\Phi_{a}$, obtaining $\Phi^{+}(\gamma)$ and $\Delta^{+}(\gamma)$ as in $\S 2$. If $\alpha \in \Delta^{+}(\gamma)$, put $\lambda_{\alpha}=$ fundamental weight dual to $\alpha$. Set $c=2(\gamma \mid \alpha) /(\alpha \mid \alpha)$ and observe that $\gamma-c \lambda_{\alpha}$ is $\Phi^{+}(\gamma)$-dominant and $\left(\Delta^{+}(\gamma) \backslash\{\alpha\}\right)$-regular. Put

$$
\psi_{\alpha}=\psi_{\gamma-c \lambda_{\alpha}}^{\gamma} \text { and } \phi_{\alpha}=\psi_{\gamma}^{\gamma-c \lambda_{\alpha}} \text {. }
$$

In view of (3.2), this always makes sense. 
Now, suppose that $V$ is an irreducible Harish-Chandra module. We know [19]: $\phi_{\alpha} \psi_{\alpha}(V)=0$ if and only if $\psi_{\alpha}(V)=0$. This leads to the $\tau$-invariant of $V$ :

$$
\tau(V)=\left\{\alpha \in \Delta^{+}(\gamma): \psi_{\alpha}(V)=0\right\}
$$

D. Vogan has carried out an extensive investigation of $\tau(\bar{\pi}(\gamma)), \gamma \in \hat{H}_{a}$. We recall two consequences of this work.

(3.5) Theorem (Vogan [13; 12, (3.2)]). Fix $\bar{\pi}(\gamma), \gamma \in \hat{H}_{a}$. Define translation functors and $\tau$-invariants relative to $\Phi^{+}(\gamma)$ and $\Delta^{+}(\gamma)$. Then $\tau(\bar{\pi}(\gamma))$ consists of those $\alpha \in \Delta^{+}(\gamma)$ which satisfy either:

(a) $\alpha$ is a real root, or

(b) $\alpha$ is a complex root and $\theta \alpha \notin \Delta^{+}(\gamma)$, or

(c) $\alpha$ is compact imaginary.

(3.6) Theorem (Vogan [2, (7.5); 17, (8.2.7)]). We define translation functors and $\tau$-invariants relative to $\Psi$, recall (2.3). Fix a standard representation $\pi(\gamma), \gamma \in \hat{H}$, and let $\phi_{\gamma}$ be the restriction of the dual of an inner automorphism of $\mathfrak{g}$, mapping $\mathfrak{b}$ into $\mathfrak{h}$, such that $\phi_{\gamma}(\Psi)=\Phi^{+}(\gamma)$. Let $\bar{\alpha}=\phi_{\gamma}(\alpha)$ be a simple complex root in $\Phi(\gamma)$, with the property that $\theta \bar{\alpha} \notin \Phi^{+}(\gamma)$. Then $\alpha$ is not in the $\tau$-invariant of any irreducible submodule of $\pi(\gamma)$.

Using the notation of (3.6), we define the $\mathscr{P}$-invariant of $\pi(\gamma), \gamma \in \hat{H}$, by

$$
\begin{aligned}
\mathscr{S}(\pi(\gamma))=\left\{\alpha \in \Psi: \phi_{\gamma}(\alpha) \in \Delta^{+}(\gamma),\right. \\
\\
\left.\phi_{\gamma}(\alpha) \text { is complex and } \theta \phi_{\gamma}(\alpha) \notin \Phi^{+}(\gamma)\right\} .
\end{aligned}
$$

Suppose $\gamma \in \hat{H}_{a}$ and $\gamma$ is integral. Define the length of $\bar{\pi}(\gamma), \gamma \in \mathfrak{h}_{a}^{*}$, by

$$
l(\bar{\pi}(\gamma))=\frac{1}{2}\left|\left\{\alpha \in \Phi^{+}(\gamma): \theta \alpha \notin \Phi^{+}(\gamma)\right\}\right|+\frac{1}{2} \operatorname{dim} a_{a, 0}-C_{0},
$$

where $C_{0}$ equals one half the dimension of the split part of a fundamental Cartan subgroup of $G$. According to [16, (12.1)], $l(\bar{\pi}(\gamma))$ is an integer. Observe, if $\bar{\pi}(\gamma)$ is finite dimensional (resp. a discrete series), then $l(\bar{\pi}(\gamma))=\frac{1}{2} \operatorname{dim} G / K-C_{0}$ (resp. $l(\bar{\pi}(\gamma))=0)$.

Fix an irreducible Harish-Chandra module with regular integral infinitesimal character $\chi_{\gamma}$. Assume $\alpha \notin \tau(V)$, then there exists a chain complex [14]

$$
C^{*}: 0 \rightarrow V \rightarrow \phi_{\alpha} \psi_{\alpha}(V) \rightarrow V \rightarrow 0,
$$

with the property that $V$ occurs as the unique irreducible submodule and quotient of $\phi_{\alpha} \psi_{\alpha}(V)$. If $U_{\alpha}(V)$ is the cohomology of the complex $C^{*}$, then D. Vogan [15] has shown

(Kazhdan-Lusztig conjectures): In the case of regular integral infinitesimal character, $U_{\alpha}(V)$ is a completely reducible Harish-Chandra module for $G$.

We need several ramifications of (3.9), the first of which is

(3.10) Theorem (Vogan $[14,(7.2) ; 13,(3.18)])$. If $\bar{\pi}\left(\gamma^{\prime}\right)$ and $\bar{\pi}(\gamma)$ are distinct and $\gamma, \gamma^{\prime}$ are regular integral, then

$$
\operatorname{Ext}^{1}\left(\bar{\pi}(\gamma), \bar{\pi}\left(\gamma^{\prime}\right)\right)=\operatorname{Ext}^{1}\left(\bar{\pi}\left(\gamma^{\prime}\right), \bar{\pi}(\gamma)\right) \neq 0,
$$

only if $l(\bar{\pi}(\gamma)) \not \equiv l\left(\bar{\pi}\left(\gamma^{\prime}\right)\right)(\bmod 2)$ (here, Ext is in the $(\mathfrak{g}, K)$-category). 
(3.11) Fine structure notation. Determining the fine structure of $\pi(\gamma)$ is taken to mean the following: Determine all irreducible submodules and quotients of $\pi(\gamma)$, and the extensions between all remaining irreducible subquotients. In order to easily state such results, we adopt certain "diagrammatic conventions". Let $\pi(\gamma)=X_{1}+$ $X_{2}+\cdots+X_{n}$ in $\mathscr{G}$.

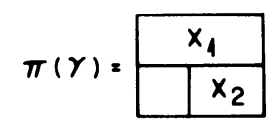

means that $X_{1}$ (resp. $\left.X_{2}\right)$ is the unique irreducible quotient (resp. submodule) of $\pi(\gamma)$ and $\operatorname{Ext}^{1}\left(X_{1}, X_{2}\right) \neq 0$;

$$
\pi(r)=\begin{array}{|l|l|l|}
\hline \multicolumn{2}{|c|}{x_{1}} \\
\hline x_{2} & \oplus & x_{3} \\
\hline
\end{array}
$$

means that $X_{1}$ is the unique irreducible quotient of $\pi(\gamma), X_{3} \oplus X_{2}$ is a submodule of $\pi(\gamma)$ and $\operatorname{Ext}^{1}\left(X_{1}, X_{i}\right) \neq 0, i=2,3$;

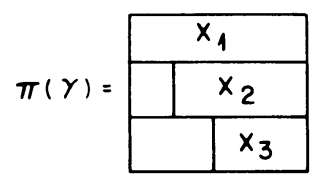

means that $X_{1}$ (resp. $X_{3}$ ) is the unique irreducible quotient (resp. submodule) of $\pi(\gamma)$ and $\operatorname{Ext}^{1}\left(X_{i}, X_{2}\right) \neq 0, i=1,3$;

$$
\pi(y)=\begin{array}{|l|l|l|}
\hline \multicolumn{3}{|c|}{x_{1}} \\
\hline x_{2} & \oplus & x_{3} \\
\hline \multicolumn{2}{|c|}{x_{4}} \\
\hline
\end{array}
$$

means that $X_{1}$ (resp. $X_{4}$ ) is the unique irreducible quotient (resp. submodule) of $\pi(\gamma), \operatorname{Ext}^{1}\left(X_{i}, X_{j}\right) \neq 0, i=1,4$ and $j=2,3$, and $X_{2} \oplus X_{3}$ is a submodule of $\pi(\gamma) / X_{4}$

(e)

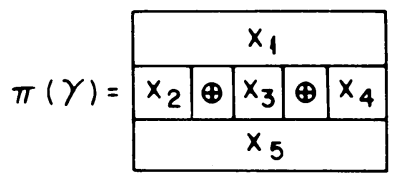

means that $X_{1}$ (resp. $X_{5}$ ) is the unique irreducible quotient (resp. submodule) of $\pi(\gamma), \operatorname{Ext}^{1}\left(X_{i}, X_{j}\right) \neq 0$ for $i=1,5$ and $j=2,3,4$, and $X_{2} \oplus X_{3} \oplus X_{4}$ is a submodule of $\pi(\gamma) / X_{5}$.

(3.12) Reduction to the case of trivial infinitesimal character. Using the translation principle [17, (8.2.1)] and (3.1), computing the fine structure of $\pi(\gamma)$ is reduced to the case $\chi_{\gamma}=\chi_{\rho}$. 
The algorithm. We now describe our procedure for computing the fine structure of $\pi(\gamma)$, in the case $\chi_{\gamma}=\chi_{\rho}$. Recall the explicit parametrizations of the standard representations: If $G=\operatorname{Spin}(2 n+1,1)$, define $\gamma_{0 i} \in \mathfrak{h}^{*}, 1 \leqslant i \leqslant n+1$, through

$$
\gamma_{0 i}=(n-i+1) e_{1}+\sum_{k=2}^{i}(n+2-k) e_{k}+\sum_{k=i+1}^{n}(n-k+1) e_{k} ;
$$

here, we let $\left\{e_{1}, e_{2}, \ldots, e_{n}\right\}$ be the standard orthonormal basis of $\mathfrak{h}^{*}$ and use the classical realization of $\Phi$, as in [4]. In all other cases, define $\gamma_{i j} \in \mathfrak{h}^{*}$ and $\gamma_{i} \in \mathfrak{b}^{*}$ as in [11], using the dictionary

$$
\begin{array}{ll}
\gamma_{i j}=\delta_{p^{i j}} & \text { as in }[11, \S 14] \\
\gamma_{i}=\delta_{(\cdots)^{\prime}} & \text { as in }[\mathbf{1 1}, \S 11],
\end{array}
$$

where $(\cdots)=A, B, C$ or $F$, according to the classification of $\Psi$.

(3.14) Theorem $[11,18]$. (i) If $G=\operatorname{Spin}(2 n+1,1)$, then $\hat{H}_{\rho}=\left\{\gamma_{0 i}: 1 \leqslant i \leqslant n+\right.$ 1) $\cup\left\{\omega_{0} \gamma_{0, n+1}\right\}$, where $\omega_{0}=$ the long element of $W(G, H)=N_{G}(H) / H$.

(ii) In all other cases, $\hat{H}_{\rho}=\left\{\gamma_{i j}:(i, j)\right.$ is given by (3.13) $\}$ and $\hat{B}_{\rho}=\left\{\gamma_{i}: i\right.$ is given by (3.13)\}.

Now, fix $G$ of the type in (3.2). Our problem is to compute the fine structure of $\pi(\gamma), \gamma \in \hat{H}_{\rho}$.

Step 1. In the case of $G=\operatorname{Spin}(2 n+1,1)$ and $\pi\left(\gamma_{0, n+1}\right)$, we have $\pi\left(\gamma_{0, n+1}\right)=$ $\bar{\pi}\left(\gamma_{0, n+1}\right)=\pi\left(\omega_{0} \gamma_{0, n+1}\right)$; cf. [18].

Step 2. Decompose $\pi(\gamma)$ in $\mathscr{G}$; this may be found among [2, 6-8 and 18]. We point out, that in the cases of $\operatorname{SP}(n, 1)$ and $F_{4}$, this decomposition is based upon David Vogan's algorithm for computing characters [13] and the Kazhdan-Lusztig conjectures (3.9).

Step 3. If $\pi(\gamma)$ has length two, then the fine structure is of type (3.11)(a), where $\bar{\pi}(\gamma)$ is the unique irreducible quotient of $\pi(\gamma)$; this uses (2.7).

Step 4. If $\pi(\gamma)$ corresponds to a character identity, then $\pi(\gamma)$ decomposes as in (3.11)(b), where $\bar{\pi}(\gamma)=X_{1}$ is the unique irreducible quotient and $X_{2}, X_{3}$ are both discrete series representations; this depends upon a well-known (unpublished) result of Wilfried Schmid [10].

Step 5. Suppose $\pi(\gamma)$ has length $\geqslant 3$ and at most one composition factor is a discrete series. Further, assume we are not in the case of $\pi\left(\gamma_{02}\right)$ or $\pi\left(\gamma_{03}\right)$ for $F_{4}$. Using (3.5)-(3.8), one explicitly computes the length and $\tau$-invariant of all composition factors and $\mathscr{S}(\pi(\gamma))$. We refer the reader to [2, $\$ 7$ and 7] for a flavor of such computations. By $(2.7), \bar{\pi}(\gamma)$ is the unique irreducible quotient of $\pi(\gamma)$ and our $\mathscr{S}$-invariant calculation will force a unique irreducible submodule. Finally, length considerations - in conjunction with (3.10) - show that all remaining subquotients have trivial extensions with one another; these are cases of the type in (3.11)(c)-(e).

Step 6. Consider the case of $\pi\left(\gamma_{02}\right)$ and $\pi\left(\gamma_{03}\right)$ for $F_{4}$. In this situation, the $\mathscr{S}$-invariant does not immediately force a unique irreducible submodule. An additional "tensoring to the wall" argument must be employed; cf. [17, (8.2.7)]. 
4. The fine structures. Fix $G$ as in (3.2) and recall the parametrizations of (3.14). Fix a regular integral infinitesimal character $\chi$, which leads us, in the obvious manner, to $\pi_{i j}(\chi), \bar{\pi}_{i j}(\chi)$ and $\pi_{i}(\chi)$. Applying the algorithm of $\S 3$, we obtain the following results; the verification of which are left to the reader.

(4.1) TheOREM. (i) If $G=\operatorname{Spin}(2 n+1,1)$, then $\pi_{0 i}(\chi)$ has fine structure:

$$
\pi_{0 i}(x)=\frac{\bar{\pi}_{0 i}(x)}{(1 \leq i \leq n)}, \pi_{0, i+1}(x), 1(x)=\bar{\pi}_{0, n+1}(x) .
$$

(ii) If $G=\operatorname{Spin}(2 n, 1)$, then $\pi_{0 i}(\chi)$ has fine structure:

$$
\begin{array}{|l|l|}
\hline & \bar{\pi}_{0 i}(x) \\
(1 \leq i \leq n-1) & \bar{\pi}_{0, i+1}(x) \\
\hline
\end{array}, \pi_{0, n}(x)=\begin{array}{|l|l|l|}
\hline \multicolumn{3}{|c|}{\bar{\pi}_{\text {on }}(x)} \\
\hline \pi_{0}(x) & \oplus & \pi_{1}(x) \\
\hline
\end{array} .
$$

(4.2) THEOREM. If $G=\mathrm{SU}(n, 1)$, then

(i) for $0 \leqslant i \leqslant n-1$,

$$
\pi_{i, n-i}(x)=\begin{array}{|l|l|l|}
\hline \multicolumn{3}{|c|}{\bar{\pi}_{i, n-i}(x)} \\
\hline \pi_{i}(x) & \oplus & \pi_{i+1}(x) \\
\hline
\end{array}
$$

(ii) for $0 \leqslant i \leqslant n-2,1 \leqslant j \leqslant n-i-1$ and $i+j<n-1$,

$$
\pi_{i, j}(x)=\begin{array}{|l|l|l|}
\hline \multicolumn{2}{|c|}{\bar{\pi}_{i j}(x)} \\
\hline \bar{\pi}_{i+1, j}(x) & \oplus & \bar{\pi}_{i, j+1}(x) \\
\hline \multicolumn{2}{|c|}{\bar{\pi}_{i+1, j+1}(x)} \\
\hline
\end{array}
$$

(iii) for $0 \leqslant i \leqslant n-2,1 \leqslant j \leqslant n-i-1$ and $i+j=n-1$,

$$
\pi_{i, j}(x)=\begin{array}{|l|l|l|}
\hline \multicolumn{3}{|c|}{\bar{\pi}_{i j}(x)} \\
\hline \bar{\pi}_{i+1, j}(x) & \oplus & \bar{\pi}_{i, j+1}(x) \\
\hline \multicolumn{3}{|c|}{\pi_{i+1}(x)} \\
\hline \multicolumn{2}{|c|}{(x)} \\
\hline
\end{array}
$$

(4.3) THEOREM. If $G=\operatorname{SP}(n, 1)$, then

(i) for $0 \leqslant i \leqslant n-1$,

$$
\pi_{i, 2 n-i}(x)=\begin{array}{|l|l|l|}
\hline \multicolumn{2}{|c|}{\bar{\pi}_{i, 2 n-i}(x)} \\
\hline \pi_{i}(x) & \oplus & \pi_{i+1}(x) \\
\hline
\end{array}
$$


(ii) for $0 \leqslant i \leqslant n-2$,

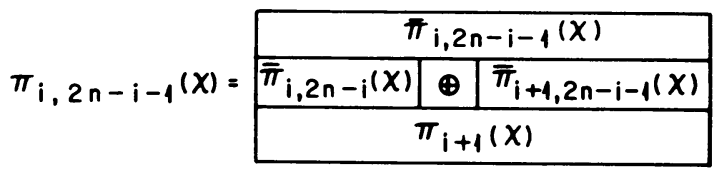

(iii)

$$
\pi_{n-1, n}(x)=\frac{\bar{\pi}_{n-1, n}(x)}{\bar{\pi}_{n-1, n+1}(x)}
$$

(iv)

$$
\pi_{n-2, n}(x)=\begin{array}{|l|l|l|l|l|}
\hline \multicolumn{4}{|c|}{\bar{\pi}_{n-2, n}(x)} \\
\hline \bar{\pi}_{n-2, n+1}(x) & \oplus & \bar{\pi}_{n-1, n}(x) & \oplus & \pi_{n}(x) \\
\hline \multicolumn{4}{|c|}{\bar{\pi}_{n-1, n+1}(x)} \\
\hline
\end{array}
$$

(v)

$$
\pi_{n-2, n-1}(x)=\begin{array}{|r|r|}
\hline & \bar{\pi}_{n-2, n-1}(x) \\
\hline & \bar{\pi}_{n-2, n}(x) \\
\hline & \pi_{n}(x) \\
\hline
\end{array}
$$

Now, assume that $0 \leqslant i \leqslant n-3, i+1 \leqslant j \leqslant 2 n-i-2$ and set $k=i+j$.

(vi) If $k$ is odd and $0 \leqslant i \leqslant[(k-1) / 2]-1$, or if $k$ is even and $0 \leqslant i \leqslant(k / 2)-2$,

$$
\pi_{i j}(x)=\begin{array}{|c|c|c|}
\hline \multicolumn{3}{|c|}{\bar{\pi}_{i j}(x)} \\
\hline \bar{\pi}_{i+1, j}(x) & \oplus & \bar{\pi}_{i, j+1}(x) \\
\hline \multicolumn{2}{|c|}{\bar{\pi}_{i+1, j+1}(x)} \\
\hline
\end{array}
$$

(vii) if $k$ is odd and $i=(k-1) / 2$, then

$$
\pi_{i j}(x)=\begin{array}{|l|l|}
\hline \multicolumn{1}{|c|}{\bar{\pi}_{i j}(x)} \\
\hline & \bar{\pi}_{i, j+1}(x) \\
\hline & \bar{\pi}_{i+2, j+2}(x) \\
\hline
\end{array}
$$

(viii) if $k$ is even and $i=(k / 2)-1$, then

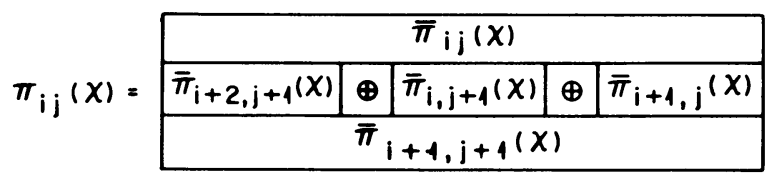


(4.4) THEOREM. If $G=F_{4}$, then

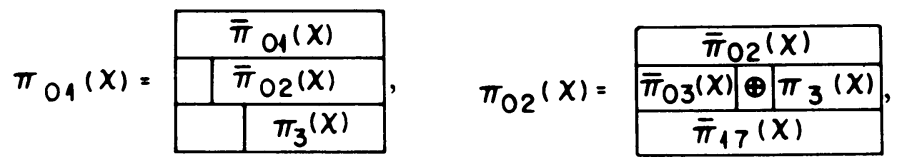

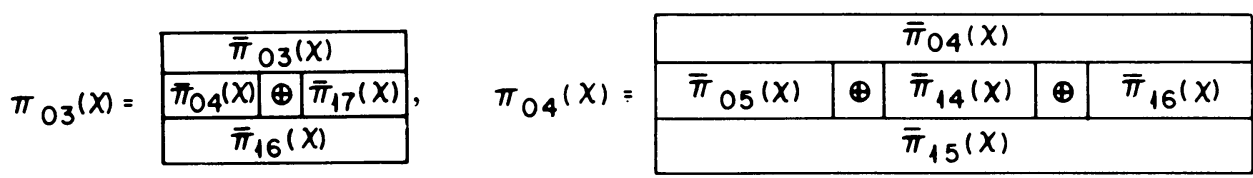

$$
\pi_{05}(x)=\begin{array}{|c|c|}
\hline \multicolumn{1}{|c|}{\bar{\pi}_{05}(x)} \\
\hline & \bar{\pi}_{15}(x) \\
\hline & \pi_{1}(x) \\
\hline
\end{array}, \quad \pi_{16}(x)=\begin{array}{|c|c|}
\hline \pi_{16}(x) \\
\hline \bar{\pi}_{17}(x) \mid \oplus \bar{\pi}_{26}(x) \\
\hline \pi_{2}(x) \\
\hline
\end{array}
$$

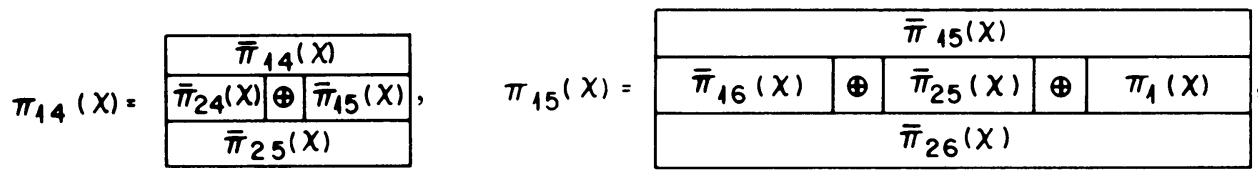

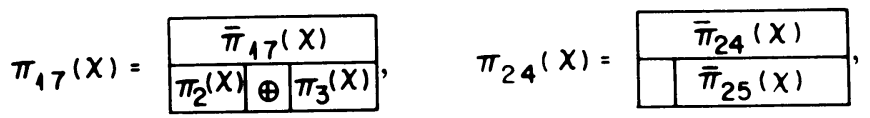

$$
\begin{aligned}
& \pi_{25}(x)=\begin{array}{|c|}
\hline \bar{\pi}_{25}(x) \\
\hline \bar{\pi}_{26}(x)
\end{array}, \quad \pi_{26}(x)=\begin{array}{|c|}
\hline \bar{\pi}_{26}(x) \\
\hline \pi_{1}(x) \oplus \pi_{2}(x) \\
\hline
\end{array} .
\end{aligned}
$$

5. The embedding lattices. Given a standard representation $\pi(\gamma)=I_{P}^{G}\left(U_{\gamma}\right)$ (recall (2.6)), then either $\pi(\gamma)$ is irreducible, or $\pi(\gamma)$ has the fine structure of its composition series determined by (4.1)-(4.4). Now, suppose the principal series representation $I_{P}^{G}\left(W \otimes e^{\nu}\right)$ has regular infinitesimal character and $\operatorname{Re}(\nu \mid \alpha) \geqslant 0$ for all $\alpha \in \Sigma^{+}$, then its fine structure is also known; since $W \otimes e^{\nu}=U_{\gamma}$, for some $\gamma$. By taking contragredients and using the fact $G$ has real rank one, we obtain

(5.1) Fine structures. The fine structure of the composition series of any principal series representation, with regular infinitesimal character, is known.

(a) If $I_{P}^{G}\left(W \otimes e^{\nu}\right)$ has nonintegral infinitesimal character, then $I_{P}^{G}\left(W \otimes e^{\nu}\right)$ is irreducible; recall (3.1).

(b) If $I_{P}^{G}\left(W \otimes e^{\nu}\right)$ satisfies $\operatorname{Re}(\nu \mid \alpha)>0$ for all $\alpha \in \Sigma^{+}$, then the fine structure follows from (4.1)-(4.4).

(c) If $I_{P}^{G}\left(W \otimes e^{\nu}\right)$ satisfies $\operatorname{Re}(\nu \mid \alpha)<0$ for all $\alpha \in \Sigma^{+}$, then the fine structure is an "upside down version" of the fine structure for some $\pi(\gamma)$; where $\gamma$ may be taken to correspond to $\omega_{0}\left(U_{\gamma}\right), \omega_{0}=$ long element of $W(G, H)$. 


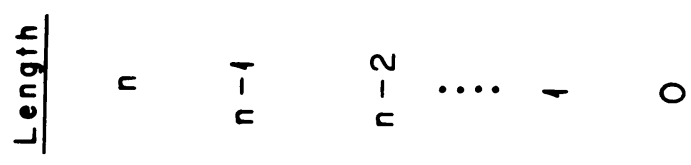

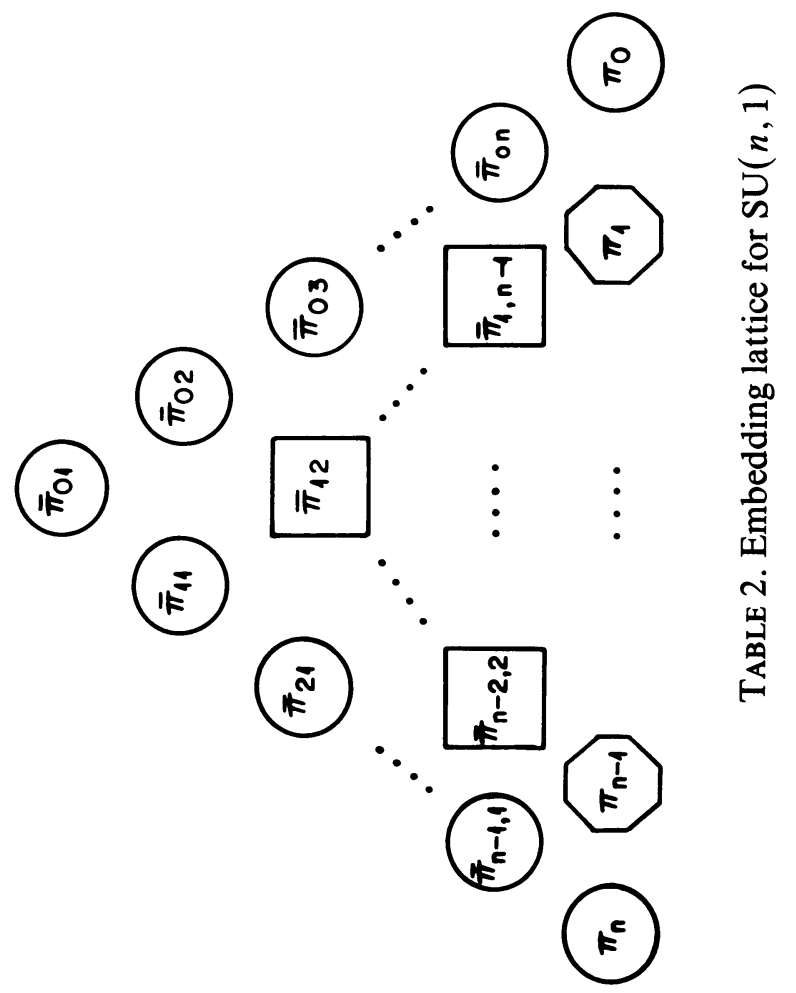

(1) 


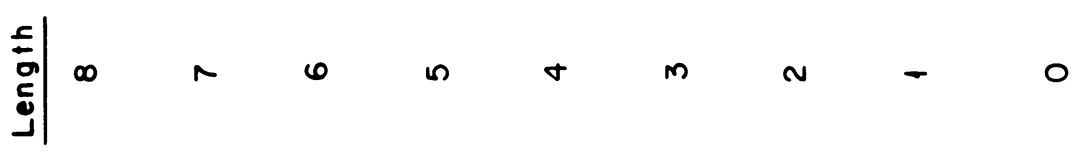

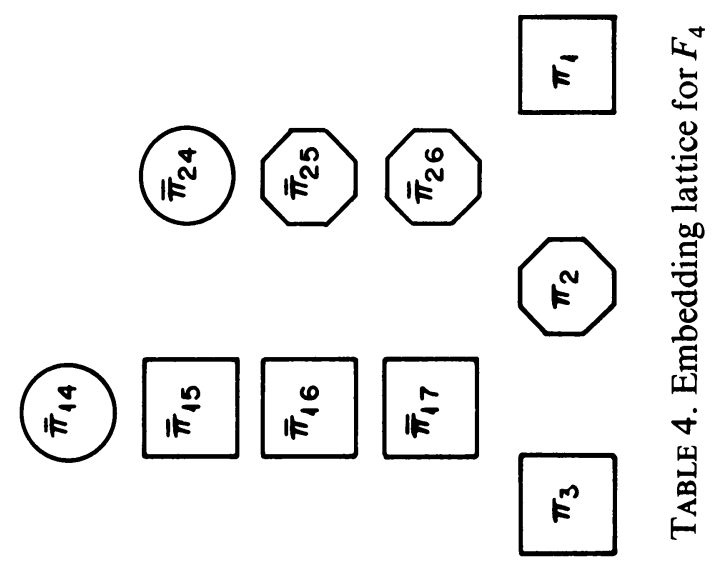

(1) 12010

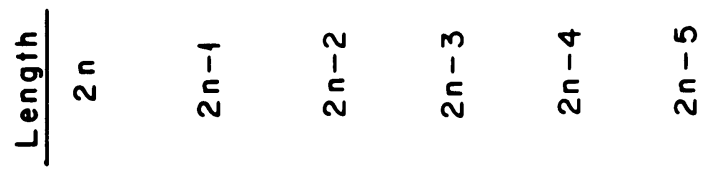

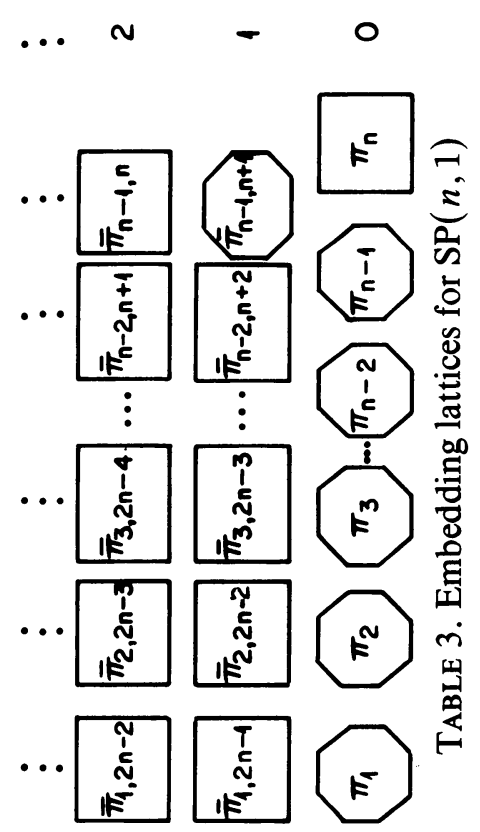

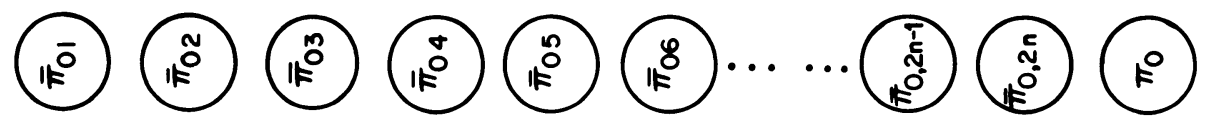


(d) If $I_{P}^{G}\left(W \otimes e^{\nu}\right)$ satisifes $\operatorname{Re}(\nu \mid \alpha)=0$ for some $\alpha \in \Sigma$, then $I_{P}^{G}\left(W \otimes e^{\nu}\right)$ is irreducible. (Note: this can only occur in the case where there is no real $\Phi$ root, i.e. when $G=\operatorname{Spin}(2 n+1,1)$.)

Using the observations in $(5.1)(a)-(d)$, it is an easy matter to compute the "number of embeddings" of a given irreducible, into principal series. To be precise, let $V$ be an irreducible Harish-Chandra module for $G$. An embedding of $V$ into principal series is taken to mean an (equivalence class of an) irreducible $M A$-module $W \otimes e^{\nu}$, which has the property $V \hookrightarrow I_{P}^{G}\left(W \otimes e^{\nu}\right)$. With this terminology, (5.1) leads us to

(5.2) Computation of embeddings. Fix an irreducible Harish-Chandra module $V$ for $G$, with regular infinitesimal character $\chi$. If $\chi$ is nonintegral, then (3.1) and (1.3) show that $V$ is an irreducible principal series representation; hence, has precisely two embeddings into principal series. Suppose $\chi$ is integral and put $V=\bar{\pi}(\gamma)$. All embeddings of $V$ into principal series are computed as follows:

(a) If $V$ is a discrete series representation, the embeddings correspond to those standard representations $\pi\left(\gamma^{\prime}\right), \gamma^{\prime} \in \hat{H}$, such that $V \hookrightarrow \pi\left(\gamma^{\prime}\right)$.

(b) If $V$ is not a discrete series representation, we always have an embedding $V \hookrightarrow I_{P}^{G}\left(\omega_{0}\left(U_{\gamma}\right)\right)$; with $U_{\gamma}$ as in (2.6) and $\omega_{0}$ as in (5.1).

(c) Determine those standard representations $\pi\left(\gamma^{\prime}\right), \gamma^{\prime} \in \hat{H}$ (if any), having the property $V \hookrightarrow \pi\left(\gamma^{\prime}\right)$.

(5.3) The embedding lattices. For each $G$ of the type in (3.2), we list all elements of

$$
\mathscr{F}_{\rho}=\left\{\bar{\pi}(\gamma): \chi_{\gamma}=\chi_{\rho}\right\}
$$

conforming to the parametrizations given in $\$ 3$ and sympathetic to the decreasing filtration given by length (3.8). We enclose $\bar{\pi}(\gamma)$ inside a circle (resp. square, octagon) as $\bar{\pi}(\gamma)$ has precisely one (resp. two, three) embeddings into principal series.

\section{REFERENCES}

1. W. Casselman, Jacquet modules for real reductive groups, Proc. Internat. Congr. Math., Helsinki, 1978, pp. 557-563.

2. D. Collingwood, Harish-Chandra modules with the unique embedding property, Trans. Amer. Math. Soc. 281 (1984), 1-48.

3. H. Hecht and W. Schmid, Characters, asymptotics and n-homology of Harish-Chandra modules, Acta Math. 151 (1983), 49-151.

4. S. Helgason, Differential geometry, Lie groups and symmetric spaces, Academic Press, New York, 1978.

5. A. Knapp, Weyl group of a cuspidal parabolic, Ann. Sci. École Norm. Sup. (4) 8 (1975).

6. H. Kraljevic, On the representations of the group $S U(n, 1)$, Trans. Amer. Math. Soc. 221 (1976),

7. H. Kraljevic and W. B. Silva, Composition factors ofthe principal series representations of the group $S P(n, 1)$, Trans. Amer. Math. Soc. 262 (1980), 447-471.

8. Composition factors of the principal series representations of the group $F_{4}$, unpublished notes.

9. D. Miličić, Asympotic hehavior of the matrix coefficients of the discrete series, Duke Math. J. 44 (1977), 59-88.

10. W. Schmid, Lecture at the Institute for Advanced Study, Princeton, N. J., February 10, 1976.

11. W. Silva, The embedddings of the discrete series in the principal series for semisimple Lie groups of real rank one, Trans. Amer. Math. Soc. 261 (1980), 303-368.

12. B. Speh and D. Vogan, Reducibility of generalized principal series representations, Acta Math. 145 (1980), 227-299. 
13. D. Vogan, Irreducible characters of semisimple Lie groups. I, Duke Math. J. 46 (1979), 61-108.

14. Irreducible characters of semisimple Lie groups. II: The Kazhdan-Lusztig conjectures, Duke Math. J. 46 (1979), 805-859.

15. __ Irreducible characters of semisimple Lie groups. III: Proof of the Kazhdan-Lusztig conjectures, Invent. Math. 71 (1983), 381-417.

16. Irreducible characters of semisimple Lie groups. IV: Character multiplicity duality, Duke Math. J. 49 (1982), 943-1073.

17. __ Representations of real reductive Lie groups, Progress in Mathematics, Birkhäuser, Basel, 1981.

18. D. Zelobenko, Description of the quasi-simple irreducible representations of the groups $U(n, 1)$ and $\operatorname{Spin}(n, 1)$, Math. USSR-Izv. 11 (1977), 31-50.

19. G. Zuckerman, Tensor products of finite and infinite dimensional representations of semisimple Lie groups, Ann. of Math. (2) 106 (1977), 295-308.

Department of Mathematics, Rutgers University, New Brunswick, New Jersey 08903

Current address: School of Mathematics, The Institute for Advanced Study, Princeton, New Jersey 08540 